Résumés des conférences et travaux

$144 \mid 2013$

2011-2012

\title{
Philologie romane
}

Conférences de l'année 2011-2012

Fabio Zinelli

\section{OpenEdition}

1 Journals

Édition électronique

URL : https://journals.openedition.org/ashp/1487

DOI : 10.4000/ashp.1487

ISSN : 1969-6310

Éditeur

Publications de l'École Pratique des Hautes Études

\section{Édition imprimée}

Date de publication : 1 septembre 2013

Pagination : 129-132

ISSN : 0766-0677

\section{Référence électronique}

Fabio Zinelli, "Philologie romane », Annuaire de l'École pratique des hautes études (EPHE), Section des sciences historiques et philologiques [En ligne], 144 | 2013, mis en ligne le 23 octobre 2014, consulté le 05 novembre 2021. URL : http://journals.openedition.org/ashp/1487 ; DOI : https://doi.org/10.4000/ ashp. 1487 


\title{
PHILOLOGIE ROMANE
}

\author{
Directeur d'études : M. Fabio ZinelLi
}

Programme de l'année 2011-2012 : I. Les chansonniers romans (précédé d'une introduction à l'ancien occitan). - II. De l'expansion de la langue française hors de la France au Moyen Âge : l'Italie du sud.

I. Les conférences se sont développées - en alternance toutes les semaines - selon deux axes de recherche distincts. Le premier axe porte sur la tradition manuscrite de la poésie des troubadours. Une nouvelle fois, notre enquête s'est principalement concentrée sur le chansonnier Vega Aguiló (Barcelona, Biblioteca de Catalunya, 7-8 = VeAg). Compilé dans le milieu de la chancellerie d'Alfonse le Magnanime entre 1423 et 1432, ce vaste recueil associe aux poètes catalans de la génération précédant le grand Ausiàs March, vingt-cinq chansons des principaux troubadours et plusieurs poèmes en français, dont deux de Guillaume de Machaut et la plupart de ceux d'Oton de Grandson. L'analyse des deux chansons de Folquet de Marseille copiées dans VeAg a fourni un bon point de repère pour illustrer les pratiques d'édition utilisées dans le domaine de la poésie lyrique médiévale.

Les deux chansons, Per Deu, Amors, ben saubetz veramen (BdT 155.16) et Sitot me suy trop tard apercebutz (BdT 155.21) ont été copiées aux f. 36r-37v de VeAg. Le texte de BdT 155.16 correspond à celui de la famille «languedocienne » représentée ici par les mss. CRfEJMVKp, Uc, Fa (groupe $\beta$ pour P. Squillacioti dans son édition de 1999, groupe $y$ pour S. Stronski premier éditeur de Folquet en 1910). Cette branche du stemma n'a pas été épargnée par une forte contamination, de la sorte qu'il est difficile de déterminer avec précision quels sont les rapports entre les témoins. On relève cependant un grand nombre de leçons communes à $\mathrm{VeAg}$ et au chansonnier $V$ (Venise, Biblioteca Marciana, 278), de la fin du XIII ${ }^{\mathrm{e}}$ s., copié également par un catalan. À propos du texte de BdT 155.21, nous avons constaté (comme P. Squillacioti et A. Alberni, dans les actes du colloque La tradizione della lirica nel Medioevo romanzo, 2012) que la version de VeAg était très proche de celle du chansonnier copié par le clerc auvergnat Bernart Amoros. Si les traces de ce manuscrit ont été perdues à la fin $d u X V I^{e}$ s., sa leçon est cependant connue grâce à plusieurs copies partielles, parmi lesquelles les mss. O'ls. Pour BdT 155.21, il est presque impossible de dire à quelle branche de la tradition le chansonnier de Bernart Amoros peut être rapproché. À lui tout seul, il pourrait même représenter une branche indépendante du stemma. Anna Alberni a d'ailleurs rappelé avec justesse que le chansonnier avait dû à un moment donné se trouver en Catalogne. Le planh catalan Ab lo cor trist envirollat d'esmay (BdT 461.2) a en effet été copié dans une de ses feuilles de garde. Nous en sommes informés par le copiste du ms. Firenze, Biblioteca Riccardiana, 2814, qui constitue, lui aussi, une copie partielle du chansonnier de Bernart (sigle $a^{l}$ ).

La question se pose de savoir si, dans le cas des œuvres de Folquet, le compilateur de VeAg a utilisé deux sources différentes. Deux chansons ont ainsi été transcrites à 
l'intérieur d'une petite série formée par quatre poèmes occitans. Les deux premiers, ceux de Blacasset BdT 96.11 et de Folquet BdT 155.16, ainsi que le dernier, celui d'Uc de Saint-Circ BdT 457.40, ont été transcrits à partir d'une source languedocienne. On pourrait donc supposer qu'il en a été de même pour la chanson BdT 155.21. VeAg aurait pu choisir les deux poèmes de Folquet à l'intérieur d'une source homogène où le montage de plusieurs sources distinctes avait déjà été réalisé. La première des deux chansons de Folquet, BdT 155.16, aurait pu de surcroît y occuper la première place. En effet, dans les mss CEJGMQPRSUc, c'est-à-dire les témoins de la rédaction languedocienne, elle ouvre la section consacrée aux poèmes de Folquet. Par ailleurs, dans plusieurs de ces mss., CEGQS (auxquels il faut ajouter $N W$ ), Folquet figure en position initiale, ce qui revient à lui donner la première place parmi les troubadours. Le philologue allemand G. Gröber a d'ailleurs qualifié de Folquetssammlungen de tels recueils. La représentation du troubadour devenu évêque y est emblématique du changement connu par la vie littéraire du Midi dans les années qui ont suivi la Croisade. La singularité de la chanson BdT 155.16 réside ainsi dans les reproches faits à Amour et dans l'emploi de maximes percutantes soulignant que l'essence de la vie courtoise se situe en dehors du labyrinthe des passions (voir le v. 41 "Cortesia non es als mas mesura »). De la bouche de l'évêque Folquet, ces propos semblent de toute évidence bien adaptés.

Nous avons élaboré une édition des deux chansons telles qu'on les lit dans $\mathrm{VeAg}$ avec un apparat critique à deux niveaux. Le premier niveau contient tout ce qui est le propre de $V e A g$ : le relevé de ses leçons particulières, la description de tout accident de copie ou les altérations du document. Le deuxième niveau, plus complexe à organiser, accueille les variantes que le chansonnier partage avec d'autres témoins ou familles de manuscrits ainsi qu'un choix de variantes significatives de la tradition textuelle. Si un tel système s'impose pour la plupart des poèmes des troubadours, seul le premier niveau de l'apparat accompagne plusieurs des poètes catalans pour lesquels $\mathrm{VeAg}$ est l'unique témoin. L'édition est également accompagnée d'un commentaire où une attention particulière a été réservée à la langue du copiste, responsable de l'introduction de nombreux catalanismes. L'édition et les commentaires seront d'ailleurs prochainement mis en ligne dans le cadre du projet d'édition électronique du chansonnier mené en collaboration avec Anna Alberni. Le projet, qui a comme siège l'université de Barcelone (IRCVM-Departament de Filologia Catalana), a été rendu possible par un Independent Starting Grant de l'European Research Council (StG-ERC 241070), dont est titulaire la même Anna Alberni.

II. Le deuxième axe concerne l'expansion de la langue et de la littérature française en Italie au Moyen Âge à l'époque de la première dynastie angevine, et plus particulièrement dans le royaume de Naples. Ce domaine est encore susceptible de réserver quelques surprises. Au regard des nombreux manuscrits français copiés en Toscane et dans le Nord de l'Italie, il est étonnant de relever aussi peu de témoins localisés à Naples. Nous savons par ailleurs que des scribes et des miniaturistes français étaient actifs à Naples, y compris en dehors de la cours angevine. Ce constat expliquerait-il en partie notre fréquente incapacité à reconnaître en tant que « napolitains » des mss. dont l'aspect extérieur nous paraît entièrement français? D'ailleurs, seul le ms. BNF, 
fr. 688 , contenant un ensemble de traductions de textes historiques (dont l'Histoire des Normands par Aimé du Mont-Cassin, récemment rééditée par M. Guéret-Laferté, Paris, 2011), est écrit dans un français truffé de formes italiennes méridionales. Son état linguistique « mixte » rappelle ainsi le mélange plus ou moins grammaticalisé de français et de formes dialectales du Nord de l'Italie dont le franco-italien « classique » fournit de nombreux exemples. Le ms. BNF, fr. 688 constitue cependant un cas exceptionnel. Une vingtaine d'autres manuscrits copiés par des scribes italiens - parmi lesquels on compte des témoins du Tristan en prose, du cycle du Méliadus / Guiron et de l'Histoire ancienne jusqu'à César - ont été considérés tour à tour par les philologues et par les historiens de l'art comme susceptibles d'avoir été copiés à Naples. De telles localisations n'ont pas toujours fait l'unanimité. Le but de notre enquête a été d'examiner le plus grand nombre de « dossiers » pour parvenir à une meilleure connaissance de la circulation et de la production des mss. en langue d'oïl dans l'Italie du Sud.

Lors des conférences de l'année 2011-2012, nous nous sommes d'abord penchés sur la traduction des Lettres à Lucilius de Sénèque exécutée pour Bartolomeo Siginulfo, « conte de Caserte », entre 1308 et 1310 . Nous avons ensuite abordé la lecture des Statuts de l'ordre chevaleresque du Noud ou du Saint Esprit au Droit Désir, fondé par Louis de Tarante en 1352 sur le modèle de l'Ordre de l'Estoile institué par Jean le Bon en 1351. Ce texte a été transmis par un seul témoin, le ms. BNF, fr. 4274, très richement décoré dans l'atelier de l'enlumineur napolitain Cristoforo Orimina. Dans la décoration du Tristan, texte copié dans le ms. BNF fr. 756-757 datable du milieu du XIV ${ }^{\mathrm{e}} \mathrm{s}$. et probablement issu d'un milieu napolitain, la forte présence du motif du nœud de Salomon a permis d'émettre l'hypothèse que ce ms. avait été exécuté pour un membre de la noblesse napolitaine appartenant à l'ordre du nœud. Le chroniqueur Matteo Villani souligne en effet que les membres portaient « nel petto uno nodo Salamone » (III, § 83) lors de la fête annuelle de l'Ordre. La forme du nœud telle qu'on la voit dans le ms. BNF, fr. 4274 est pourtant différente de celle que l'on trouve dans le ms. BNF, fr. 756-757.

Nous avons ensuite abordé l'étude des traductions contenues dans le ms. BNF, fr. 688. La datation la plus probable du ms. nous ramène au milieu du $\mathrm{XIV}^{\mathrm{e}} \mathrm{s}$. Une telle datation n'est pas contredite par l'identification par J. Kujawiński (dans Bollettino dell'Istituto Storico Italiano per il Medioevo, 102, 2010) du mystérieux comte de Militree, dédicataire des traductions, avec Ruggero de Sanseverino, comte de Mileto en Calabre, un fidèle de Robert d'Anjou (qui régna entre 1309-1343). Il en est de même pour les citations de la Commedia de Dante à l'intérieur du texte. En effet, les traces d'une diffusion de l'œuvre du grand florentin dans l'Italie du sud précèdent de peu la moitié du siècle. Les passages en question sont deux gloses : celle identifiée par M. Gasperoni et S. Maffei (dans Quaderni Francofonia, 30, 1996) et concernant Cléopâtre (« de ceste parle lo Dente par exemple de luxure » [=Enfer, V], Historia Romana, VII, 6) à laquelle nous ajoutons la glose « se le scriptor non erra » (Historia Romana, XIII, 21) qui semble rappeler Enfer, XXVIII 12 « come Livïo scrive, che non erra » (d'autant plus que Dante y parle des guerres qui, d'Hannibal aux victoires de Charles d'Anjou, ensanglantèrent au fil des siècles l'Italie du Sud). Nos conférences se sont terminées sur une des nombreuses interrogations suscitées par ce texte et qui restent sans réponse. Il s'agit d'une glose implantée dans le passage suivant de l'Historia romana, XI, 8 : 
Quar jamaiz dui pueple de diverse foi non sont bien ensemble, et se trove l'exemple defors cest livre. Une foiz vint un borjoiz a li roy d'Arragon et lo investiga et retorna o mult de gent en sa burgoizie et fist mult de mal en Arragon quar folle cose est le fidel doner foi a lo infidel. Li fil de lo roy de Mallorque vint a lo roy Karlle quant Robert son premier filz, lequel fu puiz roy, estoit duc de Calabre. Et Karlle dist lo exemple que avoit dit de lo roy d'Arragon et non le voust recevoir. Et lo evesque qui escripst en la margyne de lo livre dist cest exemple que il fu present a lo conseil del rey Karlle.

L'anecdote est à placer après 1296, date à laquelle Robert d'Anjou devient duc de Calabre, et avant 1309, année de son couronnement. Le roi de Majorque est donc Jacques II (mort en 1311). Parmi ses enfants, les deux candidats pouvant être identifiés avec le «fil de lo roy de Mallorque » sont Sanche I (1276-1324; il épousa en 1304 Marie d'Anjou, fille de Charles II) et Ferdinand III (1278-1316). Ce dernier, fait prisonnier en Morée par Thibaud de Chepoy pour ensuite être transféré à Naples où il allait retrouver sa liberté en 1309 , nous paraît être le meilleur candidat. Très proche de sa sœur Sanche de Majorque, femme de Robert d'Anjou depuis 1304, la suite des événements devait néanmoins prouver que ses sentiments « anti-angevins » avaient toujours été les plus forts. Reste à déterminer qui était le roi d'Aragon protagoniste de l'anecdote raconté par la glose et auquel il est ici fait allusion. Il nous a semblé évident que borjoiz est une faute du copiste pour bougres (et que burgoizie est à prendre dans le sens de " communauté hérétique »). L'anecdote est en effet introduite par la considération que « jamaiz dui pueple de diverse foi non sont bien ensemble » et close par la maxime : « quar folle cose est le fidel doner foi a lo infidel ». Il serait très suggestif de penser à la dispute de quatre jours engagée en juillet 1263 devant Jacques II d'Aragon opposant un juif converti au christianisme, Pablo Christiani, à Rabbi Moïse ben Nahman (Nahmanide). Ce dernier l'emporte mais doit ensuite s'exiler (d'abord à Jérusalem, ensuite à Saint-Jean d'Acre). Les termes avec lesquels l'épisode a été relaté demeurent néanmoins trop vagues pour permettre de mettre en relation, avec certitude, les deux événements. 